\title{
Estimating Cancer Risks Due to Whole Lungs Low Dose Radiotherapy With Different Techniques for Treating COVID-19 Pneumonia
}

Amin Banaei ( $\square$ amin.banaii@modares.ac.ir)

Tarbiat Modares University Faculty of Medical Sciences https://orcid.org/0000-0003-3155-1262

Bijan Hashemi

Tarbiat Modares University Faculty of Medical Sciences

Mohsen Bakhshandeh

Shahid Beheshti University of Medical Sciences: Shaheed Beheshti University of Medical Sciences

\section{Research}

Keywords: COVID-19, Low dose radiation therapy, Cancer risk, Intensity modulated radiotherapy, 3DConformal radiotherapy

Posted Date: May 25th, 2021

DOl: https://doi.org/10.21203/rs.3.rs-539728/v1

License: (c) (i) This work is licensed under a Creative Commons Attribution 4.0 International License. Read Full License

Version of Record: A version of this preprint was published at Radiation Oncology on January 20th, 2022. See the published version at https://doi.org/10.1186/s13014-021-01971-7. 


\section{Abstract}

Background: Low dose radiotherapy (LDRT) of whole lungs with photon beams is a novel under evaluation method for treating COVID-19 pneumonia. The purpose of this study was to estimate the cancer risks induced by lung LDRT as the only radiation side effects at low doses for different radiotherapy delivery techniques.

Method: Four different radiotherapy techniques including 3D-conformal with anterior and posterior fields (3D-CRT AP-PA), 3D-conformal with 8 coplanar fields (3D-CRT 8 fields), 8 fields intensity modulated radiotherapy (IMRT), and volumetric modulated arc therapy using 2 full arcs (VMAT) were planned on the CT images of 32 COVID-19 patients. Organ average and maximum doses, and PTV dose distribution indexes were calculated and compared between different techniques. The radiation induced cancer and mortality risks were estimated and compared for the assessed techniques.

Results: In IMRT and VMAT techniques, heart (mean, and max), breast (mean, and max), and stomach (mean) doses and also maximum dose in the body were significantly lower compared to 3D-CRT techniques. The calculated conformity indexes were similar in all the techniques. However, the homogeneity indexes were lower (i.e. better) in intensity modulated techniques $(P<0.03)$ with no significant differences between IMRT and VMAT plans. Lifetime lung cancer incident risks for all the delivery techniques were statistically similar $(P>0.4)$. Cancer risks for organs located closer to lung like breast, and stomach were significantly higher in 3D-CRT techniques compared to IMRT or VMAT techniques (for a 30 years old man: $2.041 \pm 0.237 \%$ Vs $1.560 \pm 0.210 \%$; and woman: $6.624 \pm 0.548 \%$ Vs $4.963 \pm 0.462 \%)(P<0.03)$. However, 8 fields 3D-conformal had significantly lower breast cancer risk compared to the 3D-CRT AP-PA technique $(\mathrm{P}<0.01)$.

Conclusion: Although all the radiotherapy techniques had low cancer risks, the overall risks induced by IMRT and VMAT radiotherapy techniques were lower than the 3D-CRT techniques and can be used clinically in younger patients or patients having higher concerns about future cancers. Higher cancer risks except the lungs are related to breast, and stomach which must be considered for lung LDRT.

\section{Background}

Application of low dose radiotherapy (lower than $0.5 \mathrm{~Gy}$ ) with photon beams for treating viral and bacterial pneumonia has a long history backs to the 1930 s (1-3). Recent studies suggested that similar approaches could be used for treating patients suffering from COVID-19 pneumonia (4-6). There are also more than 10 registered clinical trials assessing this issue (7-16) however higher doses up to 1.5 Gy were used in some of these studies $(7,9-13,15-17)$. Whole lung radiotherapy with external photon beams at low doses $(0.5$ to $1.5 \mathrm{~Gy})$ is a novel treatment option which its effectiveness is under investigation at clinical trials phase I and II. At the time of writing this article, two of the clinical trials reported that whole lung low dose radiotherapy (LDRT) can result in improvement of COVID-19 patients having severe 
pneumonia $(7,18)$, and one study reported that whole lung LDRT failed to improve clinical outcomes in critically ill patients requiring mechanical ventilation (19).

The main mechanism proposed for lung radiotherapy was the anti-inflammatory properties of low dose radiations (20). Patients with serious COVID-19 pneumonia usually acquire acute respiratory distress syndrome (ARDS) and sequential organ failure. It was proposed that the main contributor for occurring ARDS, is "Cytokine storm" which is a high inflammatory response. This response characterized by increase in interleukins IL-2 and IL-7, and macrophage inflammatory protein (1-a). Details of this mechanism that invoke a cytokine storm were described by Mehta et al. (21). Several studies suggested low dose radiotherapy as an inhibitor for inflammatory response, however the significant inhibition effect on cytokine storm has not been proven yet (20).

Although the whole lung LDRT seems to be beneficial for COVID-19 patients, the radiation side effects, which are mainly consisted of radiation induced cancers, must be evaluated for different radiotherapy techniques. There are not any acute radiation effects for lung or other organs in the chest region at less than $7 \mathrm{~Gy}$, and lung LDRT doses $(0.5$ to $1.5 \mathrm{~Gy})$ are well below this threshold. Therefore, the radiation induced cancer risks need to be evaluated or estimated for lungs LDRT of COVID-19 patients suffering from pneumonia.

The goal of whole lung LDRT is to deliver a homogeneous dose distribution to both lung volumes while sparing the normal peripheral tissues and organs like the heart, esophagus, and breasts. However, there is not any studies reporting the normal tissue dose constraints at the dose levels of lung LDRT. There are several external radiotherapy delivery techniques like 3D-conformal, intensity modulated radiotherapy (IMRT) with fixed gantry angles, and volumetric modulated arc therapy (VMAT). There are not any studies evaluating or comparing the dosimetric or radiobiological results (i.e. cancer risks) of these delivery techniques for lung LDRT. Furthermore, there are different models for estimating the cancer risks induced by low dose radiations (22-25). BEIR VII-Phase2 (Biologic Effects of lonizing Radiation) report presents a full review of the available biological, biophysical, and epidemiological literatures and proposes the most up-to-date and comprehensive risk estimates for cancer induction from exposure to low-level ionizing radiation. The lifetime attributable risk of cancer incidence and lifetime attributable risk of cancer mortality for the various site of cancers at different exposure ages could be calculated based on the preferred model proposed by this report (22). This model estimated the risks for different organs and lifetime cancer risks regarding the sex, and age of exposure. Therefore, we used this model for evaluating the cancer risks induced by lung LDRT of COVID-19 pneumonia patients.

Regarding to our literature review, there is not any study evaluating the radiation induced cancer risks at different radiotherapy protocols for whole lung radiotherapy of COVID-19 patients, therefore, in this study, the cancer risks induced by lung LDRT were estimated using the BEIR VII-phase2 preferred model for different radiotherapy delivery techniques.

\section{Methods}


This retrospective study was performed in accordance with the relevant guidelines and regulations and the methods of this study have been approved by the National Ethics Committee. The computed tomography (CT) images of 32 patients ( 16 women and 16 men) with the mean age of 54.3 years and ranged between 32 to 74 years were used in this study without any intervention in the diagnostic or treatment procedures. All the patients that their CT images were used in this study had COVID-19 diagnosis based on clinical manifestations with a positive polymerase chain reaction of the nasopharyngeal swab, antibody test, and CT image manifestations.

The exclusion criteria were patients having a history of malignancy or heart failure, and a history of radiation therapy or surgery in chest region.

\section{Planning}

For each patient, four different delivery techniques including 3D-CRT with anterior and posterior fields (AP-PA), 3D-CRT with 8 coplanar fields, IMRT with 8 fields, and VMAT with 2 full arcs techniques were simulated on the chest CT scans (arms positioned above head) of COVID-19 patients. The lungs, heart, breasts (for women), liver, stomach, esophagus, thyroid, spinal cord, and whole body were contoured on the patient's CT images in the RayStation 8.A treatment planning software (RaySearch Laboratories, Stockholm, Sweden) under the supervision of an experienced radiologist. Furthermore, the dose distribution was calculated using the collapsed cone convolution algorithm in this software with $2 \times 2 \times 2$ $\mathrm{mm}^{3}$ dose grids. The CT image matrix consisted of $512 \star 512$ pixels with $2 \mathrm{~mm}$ slice thickness.

It must be mentioned that for the CT system (Siemens 16-slice Emotion, Siemens Healthcare GmbH, Germany) used for imaging the COVID-19 patients, the RT-smartCTQA (dose.point GmbH, Germany) phantom was used to find the relationship between the Hounsfield Unit (HU) obtained from the CT and mass/electron density of different materials. This phantom consists of different cylinder rods with known materials (Air, RW3, Acrylic, titanium, lung equivalent material, muscle, and adipose equivalent materials) positioned in a 300x300x70 mm2 RW3 cube. Hounsfield units of different materials were measured using averaging of Hounsfield values in circular ROls in a transversal slice of the phantom for each of the materials (image with titanium rod for the titanium and image without titanium for other materials). ImageJ software (National Institutes of Health, USA) was used to calculate the average HU values in assessed ROls. Finally, CT HUs were defined for planning software to enable it to use the density values calculated from HUs for dose computation.

In the 3D-CRT technique with AP-PA fields, two opposite $6 \mathrm{MV}$ photon fields (anterior-posterior and posterior-anterior fields) at the gantry angles of 0 and 180 degree obtained from Siemens Artiste linear accelerator (Siemens Healthcare $\mathrm{GmbH}$, Germany) were used for irradiating at least $95 \%$ of PTV with $95 \%$ of the prescribed dose. Anterior and posterior fields' weights were optimized to minimize hot spots. Furthermore, collimators were rotated to 90 degrees for better shielding of normal tissues around the lungs. The planning target volume (PTV) included both lungs plus a margin of $0.5 \mathrm{~cm}$ in all directions to account for respiratory motions. Many studies reported the lung displacement in different directions, from 
$2.5 \mathrm{~mm}$ in anterior-posterior up to $18.5 \mathrm{~mm}$ in superior-inferior directions $(25-29)$. Although lung volume typically changes by $10-25 \%$ (27), however, we considered a $0.5 \mathrm{~cm}$ margin for PTV. No additional dose constraints were imposed on any organ at risk. Figure 1 shows the beam eye views of treatment fields in 3D-CRT AP-PA technique using 160 leaves of a multi-leaf collimator (MLC) covering the PTV while resulted in sparing organs at risks located near lungs like the esophagus and spinal cord.

We used eight coplanar fields with the gantry angles of $0,60,90,120,180,240,270$, and 300 degrees in 3D-CRT 8fields technique. The collimator angle and PTV margins were similar to 3D-CRT AP-PA technique.

Eight fields IMRT technique with $6 \mathrm{MV}$ coplanar photon beams at the gantry angles of $0,60,90,120,180$, 240,270 , and 300 degrees were used to irradiate at least $95 \%$ of the PTV with $95 \%$ of the prescribed dose. Leaf sequences using step and shoot technique produced by Siemens 160MLC multi-leaf collimator (Siemens Healthcare, Germany) were generated for IMRT plans.

Two coplanar full arcs (180 to 179 clockwise; and 180 to 181 counter-clockwise arcs) with 6MV photon beams were used to generate VMAT plans with Siemens Artiste linear accelerator using Siemens 160MLC multi-leaf collimator (Siemens healthcare, Germany).

Inverse planning for IMRT and VMAT treatment plans, including defining the plan objectives, iterations, and final dosimetry (with collapse cone convolution algorithm) were performed in RayStation 8.A treatment planning software (RaySearch Laboratories, Stockholm, Sweden). The plan objectives for IMRT and VMAT plans were similar and were presented in Table 1 for PTV and other normal structures. It must be noted that there were not any dose constraints in the literatures for lung LDRT, therefore the constraints used for optimizing IMRT and VMAT plans were selected regarding the sparing lung peripheral radiosensitive organs and covering the lungs PTV with the prescribed dose. 
Table 1

Dosimetric objectives used for IMRT and VMAT plans optimization for PTV and healthy structures

\begin{tabular}{|c|c|c|}
\hline Organ & Objective & IMRT/VMAT \\
\hline \multirow[t]{3}{*}{ PTV (lungs $+5 \mathrm{~mm}$ margins) } & V105\% & $<5 \%$ \\
\hline & V100\% & $>=95 \%$ \\
\hline & V95\% & $>=99 \%$ \\
\hline \multirow[t]{3}{*}{ Heart } & V90\% & $<30 \%$ \\
\hline & V60\% & $<40 \%$ \\
\hline & V20\% & $<90 \%$ \\
\hline \multirow[t]{2}{*}{ Breast } & V80\% & $<5 \%$ \\
\hline & V30\% & $<20 \%$ \\
\hline \multirow[t]{2}{*}{ Esophagus } & V90\% & $<10 \%$ \\
\hline & V50\% & $<50 \%$ \\
\hline Spinal Cord & Max Dose & $<95 \%$ of prescribed dose \\
\hline Body & V105\% & $<3 c c$ \\
\hline
\end{tabular}

We evaluated $1 \mathrm{~Gy}$ to whole lung as a prescribed dose, however there are different dose prescriptions for COVID-19 Whole lung LDRT including; 0.5 Gy (in 1 fraction) (9,17), 0.7 Gy (in 1 fraction) (10), 1 Gy (in 1 or 2 fractions) $(7,9,11,13)$, and $1.5 \mathrm{~Gy}$ (in 1 fraction) (18) based on current clinical trials and reports.

\section{Organ dose calculations and cancer risks estimation}

Dose distributions and dosimetric parameters including homogeneity index $(\mathrm{HI})$, conformity index $(\mathrm{Cl})$, mean organ dose, and maximum dose of organs were compared between different delivery techniques.

For each patient, the organ doses were calculated after obtaining the dose distribution for each plan. The mean dose of the organs was used for cancer risk estimation Based on the BEIR-VII report model. This model proposed a linear no-threshold model as the most reasonable description of the relation between ionizing radiation exposure at low doses and the lifetime radiation induced cancer risks.

In this report moderating factors are considered for cancer type, gender, age at exposure, and time elapsed after exposure (22). A threshold-free linear model was used to estimate solid tumors and a quadratic linear model was used to estimate the risk of leukemia. The report uses an exponential multiple-risk estimation model of the natural risk frequency in the community. For estimating the cancer 
risk based on age at radiation time (between progressive and incremental models) a combination of progressive and incremental models has been used such that in some cancers such as thyroid, the progressive model was applied. In some other cancers such as breast cancer in women, the incremental model and the weighted mean of both methods were used to estimate cancer risk. In the expression of risk, the committee has finally presented the life attributed risks (22). These values are presented as lifetime attributable risk of cancer incidence and lifetime attributable risk of cancer mortality for the various sites of cancers at different exposure ages. These values present the additional risk of different cancers and the total risk of all cancers for ages ranging from 0 to 80 years in both sexes for a dose of 0.1 Gy per 100,000 individuals.

We evaluated the cancer incidence and mortality risks for adults ( $>20$ years old), because all the CT images used in this study belonged to adults patients.

\section{Statistical analysis}

Dosimetric parameters including mean and maximum organ doses as well as $\mathrm{HI}$ and $\mathrm{Cl}$ for PTV dose distribution (30), and estimated radiation induced cancer risks for different delivery techniques (i.e. 3DCRT AP-PA, 3D-CRT 8 fields, IMRT, and VMAT) were compared using the repeated measurements and paired t-test statistical analysis.

The level of statistical significance was set at $P<0.05$, and all the statistical tests were performed in SPSS (Statistical Package for the Social Sciences) software package, V18 (SPSS Inc., Chicago, USA).

\section{Results}

Figure 2 presented the dose distribution of whole lung LDRT with different delivery techniques and 1Gy prescription for a patient in one slice. We cannot decide which technique had better dose distribution based on one slice evaluation. However, It's obvious that the heart and chest wall (breast in females) receive higher doses (similar to lung prescribed dose) in the AP-PA technique (Fig. 2-a). In intensity modulated techniques, the heart and chest wall receive lower doses (Fig. $<$ link rid="fig2" >2</link>-c and 2d) due to modulation of irradiation for sparing these structures. Furthermore, it seems that the 8 fields 3Dconformal technique showed better sparing of heart and chest wall (or breast) compared to the AP-PA technique (Fig. 2-b).

The mean \pm standard deviation values of organ doses (mean and maximum dose), $\mathrm{HI}$ and $\mathrm{Cl}$ for different radiotherapy techniques were presented in Table 2 . These doses were used for calculating the radiation induced cancer risks. In the 3D-CRT AP-PA technique, the highest radiation doses were observed in the lung, breast (for women), and heart, which are in the range of the prescribed dose. However, in IMRT and VMAT delivery techniques, the heart and breast doses and also most of the healthy organ doses (except the thyroid dose) were lower than the 3D-CRT AP-PA technique. Regarding the statistical analysis, heart (mean, and max), breast (mean, and max), and stomach (mean) doses and also maximum dose in the body were significantly lower in IMRT and VMAT compared to 3D-CRT techniques $(P<0.04)$. For example, 
mean doses of heart and breast (for women) were $31 \%$ and $54 \%$ lower in IMRT and VMAT techniques. However, there were not any significant differences between IMRT and VMAT techniques regarding the organ doses. The 3D-CRT with 8 coplanar fields showed lower heart, and breast mean doses compared to the AP-PA technique, with no statistical differences with intensity modulated techniques $(P<0.05)$. Furthermore, the organ dose differences between males and females were small $(P>0.2)$, except the breast in all the assessed treatment delivery techniques.

The calculated $\mathrm{Cl}$ indexes were similar in all the techniques. However, the $\mathrm{HI}$ indexes were lower (i.e. better) in intensity modulated techniques $(P<0.03)$ with no significant differences between IMRT and VMAT treatment plans $(P>0.6)$.

Table 2

Calculated mean \pm standard deviation values of organ doses for different radiotherapy techniques

\begin{tabular}{|lllll|}
\hline Dosimetric Parameters & 3D-CRT AP-PA & 3D-CRT 8 fields & IMRT & VMAT \\
\hline PTV Cl & $0.94 \pm 0.06$ & $0.94 \pm 0.04$ & $0.97 \pm 0.05$ & $0.95 \pm 0.05$ \\
\hline PTV HI & $0.38 \pm 0.07$ & $0.37 \pm 0.07$ & $0.30 \pm 0.06$ & $0.27 \pm 0.04$ \\
\hline Lung mean dose (Gy) & $1.17 \pm 0.15$ & $1.23 \pm 0.18$ & $1.09 \pm 0.11$ & $1.1 \pm 0.13$ \\
\hline Lungs max dose (Gy) & $1.33 \pm 0.19$ & $1.40 \pm 0.22$ & $1.43 \pm 0.15$ & $1.27 \pm 0.17$ \\
\hline Heart mean dose (Gy) & $0.96 \pm 0.10$ & $0.77 \pm 0.09$ & $0.66 \pm 0.05$ & $0.65 \pm 0.07$ \\
\hline Heart max dose (Gy) & $1.27 \pm 0.12$ & $1.25 \pm 0.11$ & $1.08 \pm 0.08$ & $1.12 \pm 0.08$ \\
\hline Breast mean dose (Gy) & $1.22 \pm 0.15$ & $0.64 \pm 0.05$ & $0.55 \pm 0.04$ & $0.59 \pm 0.05$ \\
\hline Breast max dose (Gy) & $1.35 \pm 0.17$ & $0.95 \pm 0.07$ & $0.72 \pm 0.06$ & $0.77 \pm 0.06$ \\
\hline Liver mean dose (Gy) & $0.57 \pm 0.06$ & $0.49 \pm 0.05$ & $0.55 \pm 0.06$ & $0.57 \pm 0.07$ \\
\hline Liver max dose (Gy) & $1.24 \pm 0.11$ & $1.25 \pm 0.14$ & $1.1 \pm 0.09$ & $1.19 \pm 0.1$ \\
\hline Stomach mean dose (Gy) & $0.58 \pm 0.04$ & $0.54 \pm 0.05$ & $0.43 \pm 0.05$ & $0.45 \pm 0.05$ \\
\hline Stomach max dose (Gy) & $1.15 \pm 0.11$ & $1.2 \pm 0.10$ & $0.98 \pm 0.08$ & $1.13 \pm 0.08$ \\
\hline Thyroid mean dose (Gy) & $0.11 \pm 0.01$ & $0.14 \pm 0.02$ & $0.18 \pm 0.03$ & $0.35 \pm 0.03$ \\
\hline Thyroid max dose (Gy) & $0.28 \pm 0.03$ & $0.29 \pm 0.04$ & $0.49 \pm 0.05$ & $0.98 \pm 0.08$ \\
\hline Esophagus mean dose (Gy) & $0.35 \pm 0.03$ & $0.71 \pm 0.06$ & $0.56 \pm 0.06$ & $0.61 \pm 0.07$ \\
\hline Esophagus max dose (Gy) & $0.95 \pm 0.08$ & $1.19 \pm 0.09$ & $0.98 \pm 0.07$ & $1.08 \pm 0.08$ \\
\hline Spinal Cord mean dose (Gy) & $0.22 \pm 0.02$ & $0.59 \pm 0.05$ & $0.51 \pm 0.04$ & $0.58 \pm 0.05$ \\
\hline Spinal Cord maximum dose (Gy) & $0.92 \pm 0.10$ & $1.1 \pm 0.10$ & $1.01 \pm 0.09$ & $1.06 \pm 0.08$ \\
\hline Maximum Dose in body (Gy) & $1.35 \pm 0.11$ & $1.36 \pm 0.12$ & $1.21 \pm 0.11$ & $1.23 \pm 0.09$ \\
\hline
\end{tabular}


The Radiation induced cancer incidence risks due to whole lung low dose radiotherapy (with a prescribed dose equal to $1 \mathrm{~Gy}$ ) in different techniques for a 30 years man and woman were presented in Table 3 . The Radiation induced cancer mortality risks were also presented in Table 4.

The Radiation induced cancer and mortality risks for adult patients of different ages (20 to 80 years) and different radiotherapy techniques were presented in the appendix as tables.

Table 3

Radiation induced cancer incidence risks (mean \pm standard deviation) due to whole lung low dose radiotherapy (with a prescribed dose equal to $1 \mathrm{~Gy}$ ) in different techniques for a 30 years old man and woman. Risk values are presented as incidence probability per 100 individuals.

\begin{tabular}{|lllll|}
\hline Cancer Site & 3D-CRT AP-PA & 3D-CRT 8 fields & IMRT & VMAT \\
\hline Male & & & & \\
\hline stomach & $0.134 \pm 0.011$ & $0.151 \pm 0.014$ & $0.120 \pm 0.014$ & $0.126 \pm 0.014$ \\
\hline Liver & $0.125 \pm 0.013$ & $0.108 \pm 0.011$ & $0.121 \pm 0.013$ & $0.125 \pm 0.015$ \\
\hline lung & $1.185 \pm 0.158$ & $1.292 \pm 0.189$ & $1.144 \pm 0.116$ & $1.155 \pm 0.137$ \\
\hline other & $0.416 \pm 0.040$ & $0.356 \pm 0.038$ & $0.277 \pm 0.032$ & $0.307 \pm 0.038$ \\
\hline thyroid & $0.010 \pm 0.001$ & $0.013 \pm 0.002$ & $0.016 \pm 0.003$ & $0.031 \pm 0.003$ \\
\hline all solids & $1.864 \pm 0.220$ & $1.684 \pm 0.214$ & $1.443 \pm 0.196$ & $1.533 \pm 0.202$ \\
\hline all cancers & $2.041 \pm 0.237$ & $1.835 \pm 0.230$ & $1.560 \pm 0.210$ & $1.663 \pm 0.217$ \\
\hline Female & & & & \\
\hline stomach & $0.173 \pm 0.014$ & $0.194 \pm 0.018$ & $0.155 \pm 0.018$ & $0.162 \pm 0.018$ \\
\hline Liver & $0.057 \pm 0.006$ & $0.049 \pm 0.005$ & $0.055 \pm 0.006$ & $0.057 \pm 0.007$ \\
\hline lung & $2.831 \pm 0.363$ & $2.977 \pm 0.436$ & $2.638 \pm 0.266$ & $2.662 \pm 0.315$ \\
\hline Breast & $3.087 \pm 0.430$ & $2.226 \pm 0.127$ & $2.226 \pm 0.101$ & $1.948 \pm 0.126$ \\
\hline other & $0.435 \pm 0.041$ & $0.373 \pm 0.039$ & $0.290 \pm 0.033$ & $0.321 \pm 0.035$ \\
\hline thyroid & $0.044 \pm 0.004$ & $0.057 \pm 0.008$ & $0.074 \pm 0.012$ & $0.143 \pm 0.012$ \\
\hline all solids & $6.624 \pm 0.548$ & $5.874 \pm 0.490$ & $4.963 \pm 0.462$ & $5.053 \pm 0.535$ \\
\hline all cancers & $7.236 \pm 0.695$ & $6.417 \pm 0.602$ & $5.591 \pm 0.570$ & $5.671 \pm 0.581$ \\
\hline
\end{tabular}


Table 4

Radiation induced cancer mortality risks (mean \pm standard deviation) due to whole lung low dose radiotherapy (with a prescribed dose equal to $1 \mathrm{~Gy}$ ) in different techniques for a 30 years old man and woman. Risk values are presented as incidence probability per 100 individuals.

\begin{tabular}{|lllll|}
\hline Cancer Site & 3D-CRT AP-PA & 3D-CRT 8 fields & IMRT & VMAT \\
\hline Male & & & & \\
\hline stomach & $0.093 \pm 0.006$ & $0.086 \pm 0.008$ & $0.069 \pm 0.008$ & $0.072 \pm 0.008$ \\
\hline Liver & $0.091 \pm 0.011$ & $0.078 \pm 0.008$ & $0.088 \pm 0.010$ & $0.091 \pm 0.011$ \\
\hline lung & $1.219 \pm 0.161$ & $1.316 \pm 0.193$ & $1.166 \pm 0.118$ & $1.177 \pm 0.139$ \\
\hline other & $0.197 \pm 0.019$ & $0.169 \pm 0.018$ & $0.132 \pm 0.015$ & $0.146 \pm 0.018$ \\
\hline all solids & $1.666 \pm 0.123$ & $1.571 \pm 0.121$ & $1.444 \pm 0.099$ & $1.491 \pm 0.101$ \\
\hline all cancers & $1.800 \pm 0.146$ & $1.686 \pm 0.142$ & $1.533 \pm 0.106$ & $1.590 \pm 0.108$ \\
\hline Female & & & & \\
\hline stomach & $0.122 \pm 0.008$ & $0.113 \pm 0.011$ & $0.090 \pm 0.011$ & $0.094 \pm 0.011$ \\
\hline Liver & $0.051 \pm 0.005$ & $0.044 \pm 0.005$ & $0.049 \pm 0.005$ & $0.051 \pm 0.006$ \\
\hline lung & $2.492 \pm 0.322$ & $2.620 \pm 0.383$ & $2.322 \pm 0.234$ & $2.343 \pm 0.277$ \\
\hline Breast & $0.744 \pm 0.104$ & $0.537 \pm 0.031$ & $0.537 \pm 0.024$ & $0.470 \pm 0.031$ \\
\hline other & $0.216 \pm 0.021$ & $0.185 \pm 0.020$ & $0.144 \pm 0.016$ & $0.159 \pm 0.018$ \\
\hline all solids & $3.857 \pm 0.298$ & $3.595 \pm 0.293$ & $3.117 \pm 0.319$ & $3.342 \pm 0.283$ \\
\hline all cancers & $3.971 \pm 0.308$ & $3.822 \pm 0.303$ & $3.240 \pm 0.327$ & $3.455 \pm 0.292$ \\
\hline
\end{tabular}

Lifetime lung cancer incident risks for all the delivery techniques were statistically similar $(P>0.4)$. Cancer risks for organs located closer to the lung like breast, and stomach were significantly higher in 3D-CRT techniques in comparison with IMRT or VMAT techniques $(P<0.03)$. However, 8 fields 3D-conformal had significantly lower breast cancer risk compared to the 3D-CRT AP-PA technique $(P<0.01)$. In another hand, the thyroid had lower dose and lower cancer risks in 3D-conformal techniques $(P<0.01)$. Furthermore, the 3D-CRT technique with AP-PA fields had the lowest mean and maximum dose for thyroid and esophagus, because the primary radiations from anterior and posterior fields did not pass these structures even partially.

Radiotherapy with higher prescribed doses resulted in higher cancer risks in all the delivery techniques, because the BEIR VII-phase 2 preferred model considered a linear relationship between the organs doses and cancer risks in low doses up to 2 Gy (22). 
The total lifetime cancer incidence and mortality risks at various ages of exposure for were illustrated in figure 3 for different delivery techniques. As can be noticed, the lifetime attributed risk had higher values and also higher differences at lower ages among different techniques. The cancer risk differences among different delivery techniques were reduced with increasing the age of exposure. This figure shows that the total risks were higher for females, due to the higher probability of cancer incidence for women in cancer estimation models. Furthermore, the breasts as the women's high radiosensitive organs are located near the lungs and were irradiated fully or partially in different delivery techniques.

\section{Discussion}

We evaluated the cancer risks induced by whole lung LDRT of COVID-19 pneumonia in different techniques as the main side effect of low dose radiotherapy. There are other parameters used in evaluating the radiotherapy side effects like effective dose, biological effective dose, and organ complications. In low dose radiotherapy (total dose < 1.5Gy), there were not reported any organ complications for lung and any other structures in the chest region. Although, radiation effects on different organs could be compared by the effective dose, taking into account the relative radio-sensitivity of the organs to different radiations. However, it was shown that this parameter was unable to appropriately predict the radiobiological results for low dose radiotherapy. For example, while male and female adults had received almost the same effective dose, the induced cancer risks are completely different. Furthermore, the age at the exposure as an important factor was not considered in the effective dose. Thus, the effective dose is not recommended for epidemiological evaluations (31), and in this study, we estimated the cancer risks as a radiation side effect for whole lung LDRT

It was proposed that LDRT may induce anti-inflammatory effects and helps to reduce or prevent the cytokine storm $(6,20)$. Historical reports suggest radiation doses between 0.35 and $0.5 \mathrm{~Gy}$ for lung LDRT as an optimal dose, and higher doses may induce excessive inflammation in pneumonia patients (32). Recent studies and clinical trials showed that higher doses up to $1.5 \mathrm{~Gy}$ were effective for treating the COVID-19 pneumonia $(5,7,18)$. Although we found that the IMRT and VMAT delivery techniques lead to significantly lower radiation induced cancer risks compared to AP-PA conformal technique, but we think that the effectiveness of different LDRT dose prescriptions must be compared in a clinical trial study to find the optimum LDRT dose prescription protocol for treating COVID-19 pneumonia. Therefore, we just presented the results of LDRT with 1Gy X-ray prescribed dose for different radiation delivery techniques. Since the cancer induced risk probability relation with absorbed dose is linear in low doses, one can easily calculate the cancer risks at different LDRT doses by multiplying the estimated cancer risks in this study by the radiation dose (at Gy).

Although there are several clinical trials about the treatment effectiveness of LDRT on COVID-19 pneumonia with different dose prescriptions, however, there is a frustrating lack of detail about technical delivery techniques. We tried to use different delivery techniques and evaluating their risks to prepare appropriate data for clinicians about choosing the delivery techniques in different situations. A simple AP/PA treatment method provided relatively similar conformity indexes in comparison with more 
advanced techniques (like IMRT) and may be appropriate for initial clinical trials. But we think that other delivery techniques like 3D-conformal with higher number of fields, IMRT, and VMAT are better choices for clinical applications due to their significantly lower cancer risks, especially for younger patients. Our results showed that IMRT and VMAT had lower cancer risks compared to 3D-conformal techniques. The superiority of intensity modulated techniques may be related to their modulated radiation which could better spare the organs at risks like the breast, heart, and liver. Furthermore, these techniques could be assessed in future clinical trial designs.

In several studies (33-36), it was mentioned that intensity modulated techniques had higher low dose regions in radiotherapy and therefore, the probability of secondary cancers are higher for these techniques, although the risk of organ malfunction/dysfunction and organ doses (mean and maximum doses) are lower. This may be a little confusing regarding our results. It must be mentioned that the total dose of one radiotherapy course is much higher than the LDRT. For example, the radiation dose for treatment of locally advanced prostate cancer is usually higher than $70 \mathrm{~Gy}$ in 35 fractions and the radiation doses for treating non-small cell lung cancers at stage I-III are usually higher than $50 \mathrm{~Gy}$ in equally 2 Gy per fraction regime. Therefore, the low dose regions (regions with lower than $10 \%$ of prescription dose) have doses as high as $5 \mathrm{~Gy}$, much higher than lung LDRT. Furthermore, in high dose regions, organs complications like dysfunction may occur, and secondary cancer risks are much possible where the organ complications have lower probabilities. LDRT dose levels are extremely lower than the radiotherapy regimens (30 to 100 times lower). There are not any radiation effects for lung at less than 7 Gy X-ray doses, and lung LDRT doses $(0.5$ to $1.5 \mathrm{~Gy})$ are well below this threshold $(37,38)$. In this situation, the cancer risk is directly correlated with dose, as we showed in the results of this study.

In radiotherapy, estimation of radiation induced cancer risks can be divided into two dose regions named medium dose (region positioned between $5-50 \%$ isodose lines) and low dose regions (regions lower than the $5 \%$ of the prescription dose) (39). Radiobiological factors at cellular level including DNA mutations, cell survival, cell repair and repopulation which occur during fractionated exposures must be considered in a comprehensive model which incorporates the induction of cancers in medium dose regions. There are several models addressing these issues in the literature (40-43). However, it was shown that at low doses, different fractionation regimes have the same biological results (41). Schneider et al. proposed a model for medium to small doses, the linear no-threshold model, based on the information recorded from atomic bomb survivors (40). This model was described in details by Sánchez-Nieto et al. (39). The results of the previous studies indicate that the linear models used in radiation protection which is explained by the ICRP (25) would be suitable for cancer estimation in low dose regions (< $4 \mathrm{~Gy}$ ). We used the linear model from the BEIR-VII report (22) instead of the ICRP model [34]. The ICRP model did not consider sex and age specificities in the risk estimations, furthermore, cancer risks for different sites and organs are available in the BEIR-VII model. The Schneider model $(23,24,40)$ considers the dose distributions in the radiosensitive organs for calculating the cancer risks. However, the main focus of the Schneider model is on the doses above $1 \mathrm{~Gy}$ in radiotherapy, and we think that this model is more suitable for cancer estimation in medium dose regions. We also must mention that it is not the aim of this work to decide 
which model is the most appropriate, and it's possible to use other models for cancer estimation like ICRP or Schneider model for cancer estimation of lung LDRT in COVID-19 pneumonia patients.

As a comparison between the induced cancer risks of COVID-19 LDRT and other radiological modalities, it must be mentioned that LDRT with the dose of 0.5 to $1.5 \mathrm{~Gy}$ delivers much higher ionizing radiations to patients in comparison with diagnostic radiological examinations like computed tomography or chest radiographies. For example, lung dose of $50 \mathrm{cGy}$, corresponds to approximately 11 thorax CTs, $8 \mathrm{CT}$ scans of abdomen with the highest dose protocols, 7 cardiac CTs, 7 4DCT, and 7 whole body high-quality PET/CTs. If higher dose prescriptions is administrated for COVID-19 pneumonia treatment, the number of equivalent examinations should be increased proportionally.

The dose calculation algorithm used in this study was Collapsed Cone Convolution Superposition algorithm (CCCS). The gold standard dose calculation method for dosimetry ionizing radiation is Monte Carlo. However, for large field sizes and mega voltage X-ray energies, discrepancies in dose calculation must be lower than $4 \%$ in inhomogeneity boundaries and lower than $1 \%$ in other regions for CCCS algorithms (44). The CCCS algorithm has higher agreement with Monte Carlo in inhomogeneous regions compared to other commercial radiotherapy dosimetry algorithms (45).

\section{Conclusion}

Regarding to our results, all the radiotherapy techniques had low cancer risks, and a simple AP/PA treatment method provided relatively similar homogeneity and conformity indexes in comparison with more advanced techniques (like IMRT) and may be appropriate for initial clinical trials. The overall risks induced by IMRT and VMAT radiotherapy techniques were lower than the 3D-CRT techniques and can be used clinically in younger patients or patients having higher concerns about future cancers. Higher cancer risks except the lungs are related to breast, and stomach which must be considered for lung LDRT. Furthermore, intensity modulated methods or 3D-CRT method with a high number of fields could be an appropriate radiotherapy technique for women in which the cancer risks are higher due to irradiation of breast tissue, especially in the centers having lower setup errors and radiation uncertainties.

\section{Abbreviations}

3D-CRT: 3-dimentional- conformal radiotherapy; AP-PA: Anterior posterior-posterior anterior; IMRT: Intensity modulated radiation therapy; VMAT: Volumetric modulated arc therapy; LDRT: Low dose radiotherapy; ARDS: acute respiratory distress syndrome; BEIR: Biologic effects of ionizing radiation; $\mathrm{HU}$ : Hounsfield unit; PTV: Planning tumor volume; HI: Homogeneity index; Cl: Conformity index.

\section{Declarations}

\section{Acknowledgments}


We like to express our appreciation to Negin Azadi oncology center (Tehran, Iran) for allowing us to use their software and devices. In addition, we appreciate to all the patients permit us to use their CT images for performing this research.

\section{Authors' contributions}

Amin Banaei was responsible for the study conception, design, acquisition of data, and finalizing of the manuscript. All the authors were contributed in data analyzing and writing the manuscript draft, furthermore, all the authors read and approved the final manuscript.

\section{Funding}

This work had not any funds or grant from any institution.

\section{Availability of data and materials}

All data obtained during the current study are available from the corresponding author on reasonable request.

\section{Ethics approval and consent to participate}

This retrospective study was performed in accordance with the relevant guidelines and regulations and the methods of this study have been approved by the National Ethics Committee. All the patients (or one of his/her family member) whom their CT images were used in our study were aware of using their images in this study, and sign the consents' form.

\section{Consent for publication.}

All the authors consent to publish the manuscript in Radiation Oncology.

\section{Competing interests}

The authors declare no conflict of interest.

\section{References}

1. Hess CB, Buchwald ZS, Stokes W, Nasti TH, Switchenko JM, Weinberg BD, et al. Low-dose whole-lung radiation for COVID-19 pneumonia: planned day 7 interim analysis of a registered clinical trial. Cancer. 2020;126(23):5109-13.

2. Rousseau JP, Johnson WM, Harrell GT. The value of roentgen therapy in pneumonia which fails to respond to the sulfonamides. Radiology. 1942;38(3):281-9.

3. Scott WR. X-ray Therapy in the Treatment of Acute Pneumonia: Report Covering the Use of X-ray Therapy in the Treatment of Pneumonia at the Niagara Falls Memorial Hospital, from Oct. 1, 1937, to Sept. 30, 1938. Radiology. 1939;33(3):331-49. 
4. Venkatraman P, Sahay JJ, Maidili T, Rajan R, Pooja S. Breakthrough of COVID-19 using radiotherapy treatment modalities. Radiother Oncol. 2020;148:225-6.

5. Lara PC, Burgos J, Macias D. Low dose lung radiotherapy for COVID-19 pneumonia. The rationale for a cost-effective anti-inflammatory treatment. Clin TransI Radiat Oncol. 2020;23:27-9.

6. Kirkby C, Mackenzie M. Is low dose radiation therapy a potential treatment for COVID-19 pneumonia? Radiother Oncol. 2020;147:221.

7. ClinicalTrials.gov. National Library of Medicine. Anti-inflammatory Effect of Low-Dose Whole-Lung Radiation for COVID-19 Pneumonia (May 2021). Retrieved from https://clinicaltrials.gov/ct2/show/NCT04534790?cond=radiotherapy+covid\&draw=2\&rank=13.

8. ClinicalTrials.gov. National Library of Medicine. COVID-19 Pneumonitis Low Dose Lung Radiotherapy (COLOR-19). Retrieved from https://clinicaltrials.gov/ct2/show/NCT04377477? cond=radiotherapy+covid\&draw $=2 \&$ rank $=1$.

9. ClinicalTrials.gov. National Library of Medicine. Low Dose Pulmonary Irradiation in Patients With COVID-19 Infection of Bad Prognosis (COVRTE-19) (May 2021). Retrieved from https://clinicaltrials.gov/ct2/show/NCT04414293?cond=radiotherapy+covid\&draw=2\&rank=8.

10. ClinicalTrials.gov. National Library of Medicine. Low Dose Radiation Therapy for Covid-19 Pneumonia (May 2021). Retrieved from https://clinicaltrials.gov/ct2/show/NCT04394793? cond=radiotherapy+covid\&draw $=2 \&$ rank $=10$.

11. ClinicalTrials.gov. National Library of Medicine. Low Dose Radiation Therapy for Severe-AcuteRespiratory-Syndrome-Coronavirus-2 (SARS-CoV-2), COVID-19 (COVID-RT-01) (May 2021). Retrieved from https://clinicaltrials.gov/ct2/show/NCT04598581? cond=radiotherapy+covid\&draw $=2 \&$ rank $=12$.

12. ClinicalTrials.gov. National Library of Medicine. Low Dose Radiotherapy for COVID-19 Pneumonitis (LOWRAD-Cov19) (May 2021). Retrieved from https://clinicaltrials.gov/ct2/show/NCT04420390? cond=radiotherapy+covid\&draw $=2 \&$ rank $=2$.

13. ClinicalTrials.gov. National Library of Medicine. Low Dose Whole Lung Radiation Therapy for Patients With COVID-19 and Respiratory Compromise (VENTED) (May 2021). Retrieved from https://clinicaltrials.gov/ct2/show/NCT04427566? cond=radiotherapy+covid\&draw=2\&rank=9.

14. ClinicalTrials.gov. National Library of Medicine. Low Dose Whole Lung Radiotherapy for Older Patients With COVID-19 Pneumonitis (May 2021). Retrieved from https://clinicaltrials.gov/ct2/show/NCT04493294?cond=radiotherapy+covid\&draw=2\&rank=4.

15. ClinicalTrials.gov. National Library of Medicine. Low-Dose Radiotherapy For Patients With SARSCOV-2 (COVID-19) Pneumonia (PREVENT) (May 2021). Retrieved from https://clinicaltrials.gov/ct2/show/NCT04466683?cond=radiotherapy+covid\&draw=2\&rank=7.

16. ClinicalTrials.gov. National Library of Medicine. Lung Irradiation for COVID-19 Pneumonia (May 2021). Retrieved from https://clinicaltrials.gov/ct2/show/NCT04393948? cond=radiotherapy+covid\&draw $=2 \&$ rank $=11$. 
17. ClinicalTrials.gov. National Library of Medicine. Low Dose Lung Radiotherapy to Treat COVID-19 Pneumonia (May 2021). Retrieved from https://clinicaltrials.gov/ct2/show/NCT04572412? cond=radiotherapy+covid\&draw $=2 \&$ rank $=5$.

18. Ameri A, Ameri P, Rahnama N, Mokhtari M, Sedaghat M, Hadavand F, et al. Low-dose whole-lung irradiation for COVID-19 pneumonia: Final results of a pilot study. Int J Radiat Oncol Biol Phys. 2021;109(4):859-66.

19. Papachristofilou A, Finazzi T, Blum A, Zehnder T, Zellweger N, Lustenberger J, et al. Low dose radiation therapy for severe COVID-19 pneumonia: a randomized double-blind study. Int J Radiat Oncol Biol Phys. 2021;

20. Dhawan G, Kapoor R, Dhawan R, Singh R, Monga B, Giordano J, et al. Low dose radiation therapy as a potential life saving treatment for COVID-19-induced acute respiratory distress syndrome (ARDS). Radiother Oncol. 2020;

21. Mehta P, McAuley DF, Brown M, Sanchez E, Tattersall RS, Manson JJ. COVID-19: consider cytokine storm syndromes and immunosuppression. The lancet. 2020;395(10229):1033-4.

22. Council NR. Health risks from exposure to low levels of ionizing radiation: BEIR VII phase 2. 2006;

23. Schneider U, Sumila M, Robotka J. Site-specific dose-response relationships for cancer induction from the combined Japanese A-bomb and Hodgkin cohorts for doses relevant to radiotherapy. Theor Biol Med Model. 2011;8(1):1-21.

24. Schneider U, Zwahlen D, Ross D, Kaser-Hotz B. Estimation of radiation-induced cancer from threedimensional dose distributions: Concept of organ equivalent dose. Int J Radiat Oncol Biol Phys. 2005;61(5):1510-5.

25. Valentin J. International Commission on Radiological Protection. The 2007 recommendations of the international commission on radiological protection. Ann ICRP ICRP Publ. 2007;103:2-4.

26. Boldea V, Sarrut D, Clippe S. Lung deformation estimation with non-rigid registration for radiotherapy treatment. In: International Conference on Medical Image Computing and Computer-Assisted Intervention. Springer; 2003. p. 770-7.

27. Keall PJ, Mageras GS, Balter JM, Emery RS, Forster KM, Jiang SB, et al. The management of respiratory motion in radiation oncology report of AAPM Task Group 76 a. Med Phys. 2006;33(10):3874-900.

28. Shimizu S, Shirato H, Kagei K, Nishioka T, Bo X, Dosaka-Akita H, et al. Impact of respiratory movement on the computed tomographic images of small lung tumors in three-dimensional (3D) radiotherapy. Int J Radiat Oncol Biol Phys. 2000;46(5):1127-33.

29. Weiss E, Robertson SP, Mukhopadhyay N, Hugo GD. Tumor, lymph node, and lymph node-to-tumor displacements over a radiotherapy series: analysis of interfraction and intrafraction variations using active breathing control (ABC) in lung cancer. Int J Radiat Oncol Biol Phys. 2012;82(4):e639-45.

30. Wambersie A. ICRU report 62, prescribing, recording and reporting photon beam therapy (supplement to ICRU Report 50). ICRU News. 1999; 
31. García-Hernández T, Romero-Expósito M, Sánchez-Nieto B. Low dose radiation therapy for COVID-19: Effective dose and estimation of cancer risk. Radiother Oncol. 2020;153:289-95.

32. Calabrese EJ, Dhawan G. How radiotherapy was historically used to treat pneumonia: could it be useful today? Yale J Biol Med. 2013;86(4):555.

33. Pirzkall A, Carol MP, Pickett B, Xia P, Roach III M, Verhey LJ. The effect of beam energy and number of fields on photon-based IMRT for deep-seated targets. Int J Radiat Oncol Biol Phys. 2002;53(2):43442.

34. Pirzkall A, Lohr F, Höss A, Wannenmacher M, Debus J, Carol M. Comparison of intensity-modulated radiotherapy with conventional conformal radiotherapy for complex-shaped tumors. Int J Radiat Oncol Biol Phys. 2000;48(5):1371-80.

35. Thilmann C, Sroka-Perez G, Krempien R, Hoess A, Wannenmacher M, Debus J. Inversely planned intensity modulated radiotherapy of the breast including the internal mammary chain: a plan comparison study. Technol Cancer Res Treat. 2004;3(1):69-75.

36. Hall EJ, Wuu C-S. Radiation-induced second cancers: the impact of 3D-CRT and IMRT. Int J Radiat Oncol Biol Phys. 2003;56(1):83-8.

37. Marks LB, Yorke ED, Jackson A, Ten Haken RK, Constine LS, Eisbruch A, et al. Use of normal tissue complication probability models in the clinic. Int J Radiat Oncol Biol Phys. 2010;76(3):S10-9.

38. Trott KR, Zschaeck S, Beck M. Radiation therapy for COVID-19 pneumopathy. Radiother Oncol. 2020 Jun;147:210-1.

39. Sánchez-Nieto B, Romero-Expósito M, Terrón JA, Sánchez-Doblado F. Uncomplicated and CancerFree Control Probability (UCFCP): A new integral approach to treatment plan optimization in photon radiation therapy. Phys Med. 2017 Oct 1;42:277-84.

40. Schneider U. Mechanistic model of radiation-induced cancer after fractionated radiotherapy using the linear-quadratic formula. Med Phys. 2009;36(4):1138-43.

41. Shuryak I, Hahnfeldt P, Hlatky L, Sachs RK, Brenner DJ. A new view of radiation-induced cancer: integrating short- and long-term processes. Part II: second cancer risk estimation. Radiat Environ Biophys. 2009 Aug;48(3):275-86.

42. Daşu A, Toma-Daşu I, Olofsson J, Karlsson M. The use of risk estimation models for the induction of secondary cancers following radiotherapy. Acta Oncol Stockh Swed. 2005;44(4):339-47.

43. Sachs RK, Brenner DJ. Solid tumor risks after high doses of ionizing radiation. Proc Natl Acad Sci U S A. 2005 Sep 13;102(37):13040-5.

44. Schwarz M, Cattaneo GM, Marrazzo L. Geometrical and dosimetrical uncertainties in hypofractionated radiotherapy of the lung: A review. Phys Medica Eur J Med Phys. 2017 Apr 1;36:126-39.

45. Zhen H, Hrycushko B, Lee H, Timmerman R, Pompoš A, Stojadinovic S, et al. Dosimetric comparison of Acuros XB with collapsed cone convolution/superposition and anisotropic analytic algorithm for stereotactic ablative radiotherapy of thoracic spinal metastases. J Appl Clin Med Phys. $2015 \mathrm{Jul}$ 8;16(4):181-92. 


\section{Appendix}

The Radiation induced cancer and mortality risks for adult patients in different ages (20 to 80 years) and different radiotherapy techniques were presented in the following tables (Table 5-12).

Tables 5 and 6 represent the radiation induced cancer and mortality risks respectively, for 3D-CRT AP-PA technique.

Table 5

The mean lifetime attributed cancer induction risks due to whole lung low dose radiotherapy (with a prescribed dose equal to $1 \mathrm{~Gy}$ ) in 3D-CRT AP-PA technique for adult male and female patients at different ages. Risk values are presented as incidence probability per 100 individuals.

\begin{tabular}{|llllllll|}
\hline \multicolumn{7}{|c|}{ Age at exposure time (years) } \\
\hline & $\mathbf{2 0}$ & $\mathbf{3 0}$ & $\mathbf{4 0}$ & $\mathbf{5 0}$ & 60 & 70 & $\mathbf{8 0}$ \\
\hline male & & & & & & & \\
\hline stomach & 0.192 & 0.134 & 0.130 & 0.120 & 0.096 & 0.067 & 0.034 \\
\hline Liver & 0.171 & 0.125 & 0.120 & 0.108 & 0.080 & 0.046 & 0.017 \\
\hline lung & 1.743 & 1.785 & 1.217 & 1.182 & 1.041 & 0.761 & 0.398 \\
\hline other & 0.655 & 0.416 & 0.361 & 0.294 & 0.206 & 0.120 & 0.048 \\
\hline thyroid & 0.023 & 0.010 & 0.003 & 0.001 & 0.000 & 0.000 & 0.000 \\
\hline all solids & 2.621 & 1.791 & 1.678 & 1.508 & 1.211 & 0.803 & 0.375 \\
\hline all cancers & 2.907 & 2.041 & 1.928 & 1.758 & 1.455 & 1.021 & 0.518 \\
\hline Female & & & & & & & \\
\hline stomach & 0.250 & 0.173 & 0.168 & 0.154 & 0.130 & 0.091 & 0.053 \\
\hline Liver & 0.080 & 0.057 & 0.057 & 0.051 & 0.040 & 0.029 & 0.011 \\
\hline lung & 4.048 & 2.831 & 2.808 & 2.691 & 2.352 & 1.720 & 0.901 \\
\hline Breast & 5.234 & 3.087 & 1.720 & 0.854 & 0.378 & 0.146 & 0.049 \\
\hline other & 0.678 & 0.435 & 0.380 & 0.311 & 0.229 & 0.143 & 0.063 \\
\hline thyroid & 0.124 & 0.045 & 0.015 & 0.004 & 0.001 & 0.000 & 0.000 \\
\hline all solids & 10.701 & 6.808 & 5.599 & 4.607 & 3.594 & 2.432 & 1.203 \\
\hline all cancers & 11.184 & 7.236 & 6.020 & 5.028 & 3.981 & 2.779 & 1.454 \\
\hline
\end{tabular}


Table 6

The mean lifetime attributed cancer mortality risks due to whole lung low dose radiotherapy (with a prescribed dose equal to $1 \mathrm{~Gy}$ ) in 3D-CRT AP-PA technique for adult male and female patients at different ages. Risk values are presented as incidence probability per 100 individuals.

\begin{tabular}{|llllllll|}
\hline \multicolumn{7}{|c|}{ Age at exposure time (years) } \\
\hline & 20 & $\mathbf{3 0}$ & $\mathbf{4 0}$ & $\mathbf{5 0}$ & $\mathbf{6 0}$ & $\mathbf{7 0}$ & $\mathbf{8 0}$ \\
\hline male & & & & & & & \\
\hline stomach & 0.122 & 0.093 & 0.087 & 0.075 & 0.064 & 0.046 & 0.023 \\
\hline Liver & 0.131 & 0.091 & 0.091 & 0.080 & 0.068 & 0.046 & 0.023 \\
\hline lung & 1.767 & 1.819 & 1.252 & 1.217 & 1.088 & 0.831 & 0.491 \\
\hline other & 0.281 & 0.197 & 0.185 & 0.162 & 0.122 & 0.076 & 0.036 \\
\hline all solids & 2.098 & 1.498 & 1.465 & 1.365 & 1.162 & 0.855 & 0.482 \\
\hline all cancers & 2.414 & 1.800 & 1.781 & 1.701 & 1.507 & 1.181 & 0.723 \\
\hline Female & & & & & & & \\
\hline stomach & 0.168 & 0.122 & 0.116 & 0.110 & 0.093 & 0.075 & 0.046 \\
\hline Liver & 0.068 & 0.051 & 0.046 & 0.046 & 0.040 & 0.029 & 0.017 \\
\hline lung & 3.569 & 2.492 & 2.480 & 2.387 & 2.141 & 1.638 & 0.948 \\
\hline Breast & 1.232 & 0.744 & 0.427 & 0.232 & 0.110 & 0.061 & 0.024 \\
\hline other & 0.309 & 0.216 & 0.204 & 0.181 & 0.145 & 0.099 & 0.050 \\
\hline all solids & 5.209 & 3.597 & 3.334 & 3.041 & 2.594 & 1.942 & 1.114 \\
\hline all cancers & 5.583 & 3.971 & 3.715 & 3.436 & 2.997 & 2.323 & 1.392 \\
\hline & & & & & & & \\
\hline
\end{tabular}

Tables 7 and 8 represent the radiation induced cancer and mortality risks respectively, for 3D-CRT 8 fields technique. 
Table 7

The mean lifetime attributed cancer induction risks due to whole lung low dose radiotherapy (with a prescribed dose equal to $1 \mathrm{~Gy}$ ) in 3D-CRT 8 fields technique for adult male and female patients at different ages. Risk values are presented as incidence probability per 100 individuals.

\begin{tabular}{|llllllll|}
\hline \multicolumn{7}{|c|}{ Age at exposure time (years) } \\
\hline & $\mathbf{2 0}$ & $\mathbf{3 0}$ & $\mathbf{4 0}$ & $\mathbf{5 0}$ & $\mathbf{6 0}$ & $\mathbf{7 0}$ & $\mathbf{8 0}$ \\
\hline male & & & & & & & \\
\hline stomach & 0.216 & 0.151 & 0.146 & 0.135 & 0.108 & 0.076 & 0.038 \\
\hline Liver & 0.147 & 0.108 & 0.103 & 0.093 & 0.069 & 0.039 & 0.015 \\
\hline lung & 1.833 & 1.292 & 1.279 & 1.242 & 1.095 & 0.800 & 0.418 \\
\hline other & 0.562 & 0.356 & 0.310 & 0.252 & 0.176 & 0.103 & 0.041 \\
\hline thyroid & 0.029 & 0.013 & 0.004 & 0.001 & 0.000 & 0.000 & 0.000 \\
\hline all solids & 2.357 & 1.610 & 1.509 & 1.356 & 1.089 & 0.722 & 0.337 \\
\hline all cancers & 2.613 & 1.835 & 1.733 & 1.581 & 1.308 & 0.918 & 0.465 \\
\hline Female & & & & & & & \\
\hline stomach & 0.281 & 0.194 & 0.189 & 0.173 & 0.146 & 0.103 & 0.059 \\
\hline Liver & 0.069 & 0.049 & 0.049 & 0.044 & 0.034 & 0.025 & 0.010 \\
\hline lung & 4.256 & 2.977 & 2.952 & 2.829 & 2.472 & 1.808 & 0.947 \\
\hline Breast & 3.775 & 2.226 & 1.241 & 0.616 & 0.273 & 0.106 & 0.035 \\
\hline other & 0.581 & 0.373 & 0.326 & 0.266 & 0.196 & 0.122 & 0.054 \\
\hline thyroid & 0.158 & 0.057 & 0.020 & 0.006 & 0.001 & 0.000 & 0.000 \\
\hline all solids & 9.490 & 6.037 & 4.965 & 4.085 & 3.187 & 2.157 & 1.066 \\
\hline all cancers & 9.918 & 6.417 & 5.338 & 4.459 & 3.531 & 2.464 & 1.289 \\
\hline & & & & & & & \\
\hline
\end{tabular}


Table 8

The mean lifetime attributed cancer mortality risks due to whole lung low dose radiotherapy (with a prescribed dose equal to $1 \mathrm{~Gy}$ ) in 3D-CRT 8 fields technique for adult male and female patients at different ages. Risk values are presented as incidence probability per 100 individuals.

\begin{tabular}{|llllllll|}
\hline \multicolumn{7}{|c|}{ Age at exposure time (years) } \\
\hline & $\mathbf{2 0}$ & $\mathbf{3 0}$ & $\mathbf{4 0}$ & $\mathbf{5 0}$ & $\mathbf{6 0}$ & $\mathbf{7 0}$ & $\mathbf{8 0}$ \\
\hline male & & & & & & & \\
\hline stomach & 0.113 & 0.086 & 0.081 & 0.070 & 0.059 & 0.043 & 0.022 \\
\hline Liver & 0.113 & 0.078 & 0.078 & 0.069 & 0.059 & 0.039 & 0.020 \\
\hline lung & 1.857 & 1.316 & 1.316 & 1.279 & 1.144 & 0.873 & 0.517 \\
\hline other & 0.241 & 0.169 & 0.158 & 0.139 & 0.104 & 0.065 & 0.031 \\
\hline all solids & 1.965 & 1.403 & 1.372 & 1.279 & 1.089 & 0.801 & 0.451 \\
\hline all cancers & 2.261 & 1.686 & 1.668 & 1.593 & 1.412 & 1.106 & 0.677 \\
\hline Female & & & & & & & \\
\hline stomach & 0.157 & 0.113 & 0.108 & 0.103 & 0.086 & 0.070 & 0.043 \\
\hline Liver & 0.059 & 0.044 & 0.039 & 0.039 & 0.034 & 0.025 & 0.015 \\
\hline lung & 3.752 & 2.620 & 2.608 & 2.509 & 2.251 & 1.722 & 0.996 \\
\hline Breast & 0.889 & 0.537 & 0.308 & 0.167 & 0.079 & 0.044 & 0.018 \\
\hline other & 0.265 & 0.185 & 0.175 & 0.155 & 0.124 & 0.085 & 0.043 \\
\hline all solids & 5.014 & 3.462 & 3.209 & 2.926 & 2.496 & 1.869 & 1.072 \\
\hline all cancers & 5.373 & 3.822 & 3.575 & 3.307 & 2.884 & 2.235 & 1.340 \\
\hline & & & & & & & \\
\hline
\end{tabular}

Tables 9 and 10 represent the radiation induced cancer and mortality risks respectively, for IMRT technique. 
Table 9

The mean lifetime attributed cancer induction risks due to whole lung low dose radiotherapy (with a prescribed dose equal to $1 \mathrm{~Gy}$ ) in IMRT technique for adult male and female patients at different ages. Risk values are presented as incidence probability per 100 individuals.

\begin{tabular}{|llllllll|}
\hline \multicolumn{7}{|c|}{ Age at exposure time (years) } \\
\hline & $\mathbf{2 0}$ & $\mathbf{3 0}$ & $\mathbf{4 0}$ & $\mathbf{5 0}$ & $\mathbf{6 0}$ & $\mathbf{7 0}$ & $\mathbf{8 0}$ \\
\hline male & & & & & & & \\
\hline stomach & 0.172 & 0.120 & 0.116 & 0.108 & 0.086 & 0.060 & 0.030 \\
\hline Liver & 0.165 & 0.121 & 0.116 & 0.105 & 0.077 & 0.044 & 0.017 \\
\hline lung & 1.624 & 1.145 & 1.134 & 1.101 & 0.970 & 0.709 & 0.371 \\
\hline other & 0.437 & 0.277 & 0.241 & 0.196 & 0.137 & 0.080 & 0.032 \\
\hline thyroid & 0.038 & 0.016 & 0.005 & 0.002 & 0.001 & 0.000 & 0.000 \\
\hline all solids & 2.003 & 1.369 & 1.283 & 1.153 & 0.926 & 0.614 & 0.287 \\
\hline all cancers & 2.222 & 1.560 & 1.474 & 1.344 & 1.112 & 0.780 & 0.396 \\
\hline Female & & & & & & & \\
\hline stomach & 0.224 & 0.155 & 0.151 & 0.138 & 0.116 & 0.082 & 0.047 \\
\hline Liver & 0.077 & 0.055 & 0.055 & 0.050 & 0.039 & 0.028 & 0.011 \\
\hline lung & 3.771 & 2.638 & 2.616 & 2.507 & 2.191 & 1.602 & 0.839 \\
\hline Breast & 3.775 & 2.226 & 1.241 & 0.616 & 0.273 & 0.106 & 0.035 \\
\hline other & 0.452 & 0.290 & 0.253 & 0.207 & 0.153 & 0.095 & 0.042 \\
\hline thyroid & 0.203 & 0.074 & 0.025 & 0.007 & 0.002 & 0.001 & 0.000 \\
\hline all solids & 8.268 & 5.260 & 4.326 & 3.559 & 2.777 & 1.879 & 0.929 \\
\hline all cancers & 8.641 & 5.591 & 4.651 & 3.885 & 3.076 & 2.147 & 1.123 \\
\hline & & & & & & & \\
\hline
\end{tabular}


Table 10

The mean lifetime attributed cancer mortality risks due to whole lung low dose radiotherapy (with a prescribed dose equal to $1 \mathrm{~Gy}$ ) in IMRT technique for adult male and female patients at different ages. Risk values are presented as incidence probability per 100 individuals.

\begin{tabular}{|llllllll|}
\hline \multicolumn{7}{|c|}{ Age at exposure time (years) } \\
\hline & $\mathbf{2 0}$ & $\mathbf{3 0}$ & $\mathbf{4 0}$ & $\mathbf{5 0}$ & $\mathbf{6 0}$ & $\mathbf{7 0}$ & $\mathbf{8 0}$ \\
\hline male & & & & & & & \\
stomach & 0.090 & 0.069 & 0.065 & 0.056 & 0.047 & 0.034 & 0.017 \\
\hline Liver & 0.127 & 0.088 & 0.088 & 0.077 & 0.066 & 0.044 & 0.022 \\
\hline lung & 1.646 & 1.166 & 1.166 & 1.134 & 1.014 & 0.774 & 0.458 \\
\hline other & 0.188 & 0.132 & 0.123 & 0.108 & 0.081 & 0.050 & 0.024 \\
\hline all solids & 1.786 & 1.275 & 1.247 & 1.163 & 0.990 & 0.728 & 0.410 \\
\hline all cancers & 2.056 & 1.533 & 1.517 & 1.449 & 1.284 & 1.006 & 0.616 \\
\hline Female & & & & & & & \\
\hline stomach & 0.125 & 0.090 & 0.086 & 0.082 & 0.069 & 0.056 & 0.034 \\
\hline Liver & 0.066 & 0.050 & 0.044 & 0.044 & 0.039 & 0.028 & 0.017 \\
\hline lung & 3.325 & 2.322 & 2.311 & 2.224 & 1.995 & 1.526 & 0.883 \\
\hline Breast & 0.889 & 0.537 & 0.308 & 0.167 & 0.079 & 0.044 & 0.018 \\
\hline other & 0.206 & 0.144 & 0.136 & 0.120 & 0.097 & 0.066 & 0.034 \\
\hline all solids & 4.250 & 2.935 & 2.720 & 2.481 & 2.116 & 1.584 & 0.909 \\
\hline all cancers & 4.555 & 3.240 & 3.031 & 2.804 & 2.445 & 1.895 & 1.136 \\
\hline
\end{tabular}

Tables 11 and 12 represent the radiation induced cancer and mortality risks respectively, for VMAT technique. 
Table 11

The mean lifetime attributed cancer induction risks due to whole lung low dose radiotherapy (with a prescribed dose equal to $1 \mathrm{~Gy}$ ) in VMAT technique for adult male and female patients at different ages. Risk values are presented as incidence probability per 100 individuals.

\begin{tabular}{|llllllll|}
\hline \multicolumn{7}{|c|}{ Age at exposure time (years) } \\
\hline & 20 & 30 & 40 & 50 & 60 & 70 & 80 \\
\hline male & & & & & & & \\
\hline stomach & 0.180 & 0.126 & 0.122 & 0.113 & 0.090 & 0.063 & 0.032 \\
\hline Liver & 0.171 & 0.125 & 0.120 & 0.108 & 0.080 & 0.046 & 0.017 \\
\hline lung & 1.639 & 1.155 & 1.144 & 1.111 & 0.979 & 0.715 & 0.374 \\
\hline other & 0.484 & 0.307 & 0.267 & 0.217 & 0.152 & 0.088 & 0.036 \\
\hline thyroid & 0.074 & 0.032 & 0.011 & 0.004 & 0.001 & 0.000 & 0.000 \\
\hline all solids & 2.136 & 1.459 & 1.367 & 1.229 & 0.987 & 0.655 & 0.305 \\
\hline all cancers & 2.368 & 1.663 & 1.571 & 1.433 & 1.185 & 0.832 & 0.422 \\
\hline Female & & & & & & & \\
\hline stomach & 0.234 & 0.162 & 0.158 & 0.144 & 0.122 & 0.086 & 0.050 \\
\hline Liver & 0.080 & 0.057 & 0.057 & 0.051 & 0.040 & 0.029 & 0.011 \\
\hline lung & 3.806 & 2.662 & 2.640 & 2.530 & 2.211 & 1.617 & 0.847 \\
\hline Breast & 3.303 & 1.948 & 1.086 & 0.539 & 0.239 & 0.092 & 0.031 \\
\hline other & 0.501 & 0.321 & 0.281 & 0.229 & 0.169 & 0.105 & 0.047 \\
\hline thyroid & 0.396 & 0.144 & 0.049 & 0.014 & 0.004 & 0.001 & 0.000 \\
\hline all solids & 8.387 & 5.336 & 4.388 & 3.610 & 2.817 & 1.906 & 0.943 \\
\hline all cancers & 8.765 & 5.671 & 4.718 & 3.940 & 3.120 & 2.178 & 1.140 \\
\hline & & & & & & & \\
\hline
\end{tabular}


Table 12

The mean lifetime attributed cancer mortality risks due to whole lung low dose radiotherapy (with a prescribed dose equal to $1 \mathrm{~Gy}$ ) in VMAT technique for adult male and female patients at different ages. Risk values are presented as incidence probability per 100 individuals

\begin{tabular}{|llllllll|}
\hline \multicolumn{7}{|c|}{ Age at exposure time (years) } \\
\hline & $\mathbf{2 0}$ & $\mathbf{3 0}$ & $\mathbf{4 0}$ & $\mathbf{5 0}$ & $\mathbf{6 0}$ & $\mathbf{7 0}$ & $\mathbf{8 0}$ \\
\hline male & & & & & & & \\
\hline stomach & 0.095 & 0.072 & 0.068 & 0.059 & 0.050 & 0.036 & 0.018 \\
\hline Liver & 0.131 & 0.091 & 0.091 & 0.080 & 0.068 & 0.046 & 0.023 \\
\hline lung & 1.661 & 1.177 & 1.177 & 1.144 & 1.023 & 0.781 & 0.462 \\
\hline other & 0.208 & 0.146 & 0.136 & 0.119 & 0.090 & 0.056 & 0.026 \\
\hline all solids & 1.853 & 1.323 & 1.294 & 1.206 & 1.027 & 0.755 & 0.426 \\
\hline leukemia & 0.280 & 0.267 & 0.280 & 0.296 & 0.305 & 0.288 & 0.213 \\
\hline all cancers & 2.133 & 1.590 & 1.573 & 1.502 & 1.331 & 1.043 & 0.639 \\
\hline Female & & & & & & & \\
\hline stomach & 0.131 & 0.095 & 0.090 & 0.086 & 0.072 & 0.059 & 0.036 \\
\hline Liver & 0.068 & 0.051 & 0.046 & 0.046 & 0.040 & 0.029 & 0.017 \\
\hline lung & 3.355 & 2.343 & 2.332 & 2.244 & 2.013 & 1.540 & 0.891 \\
\hline Breast & 0.778 & 0.470 & 0.270 & 0.146 & 0.069 & 0.039 & 0.015 \\
\hline other & 0.228 & 0.160 & 0.150 & 0.133 & 0.107 & 0.073 & 0.037 \\
\hline all solids & 4.532 & 3.130 & 2.900 & 2.645 & 2.257 & 1.689 & 0.969 \\
\hline all cancers & 4.857 & 3.455 & 3.232 & 2.990 & 2.607 & 2.021 & 1.211 \\
\hline
\end{tabular}

\section{Figures}



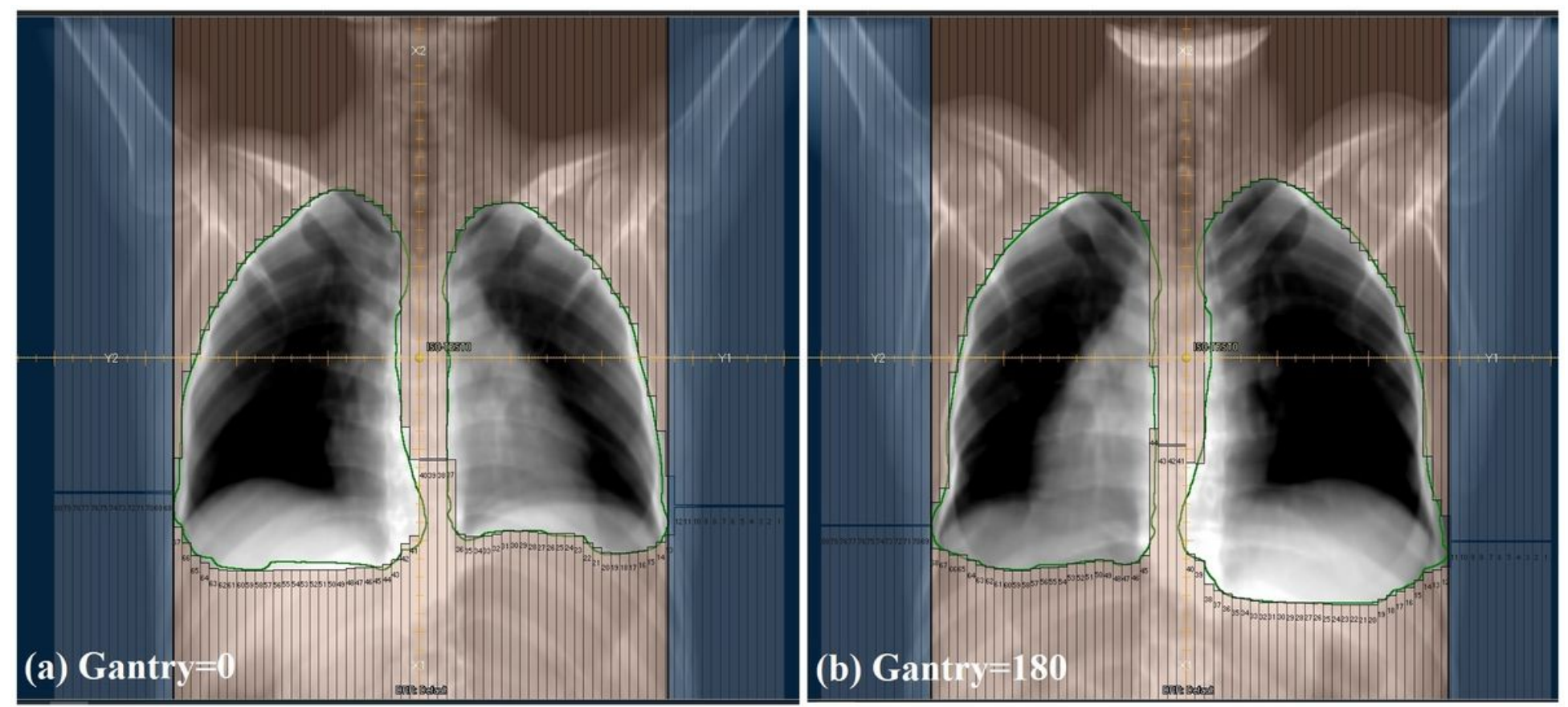

\section{Figure 1}

The beam eye views of treatment fields in 3D-CRT AP-PA technique using 160 leaves of MLC covering the PTV (specified by the green line) and sparing organs at risks located near lungs. The collimator rotated 90 degrees for better sparing of peripheral structures. (a): Beam eye view of the anterior to posterior (AP) field (gantry angle $=0$ ). (b): Beam eye view of the posterior to anterior (PA) field (gantry angle = 180).
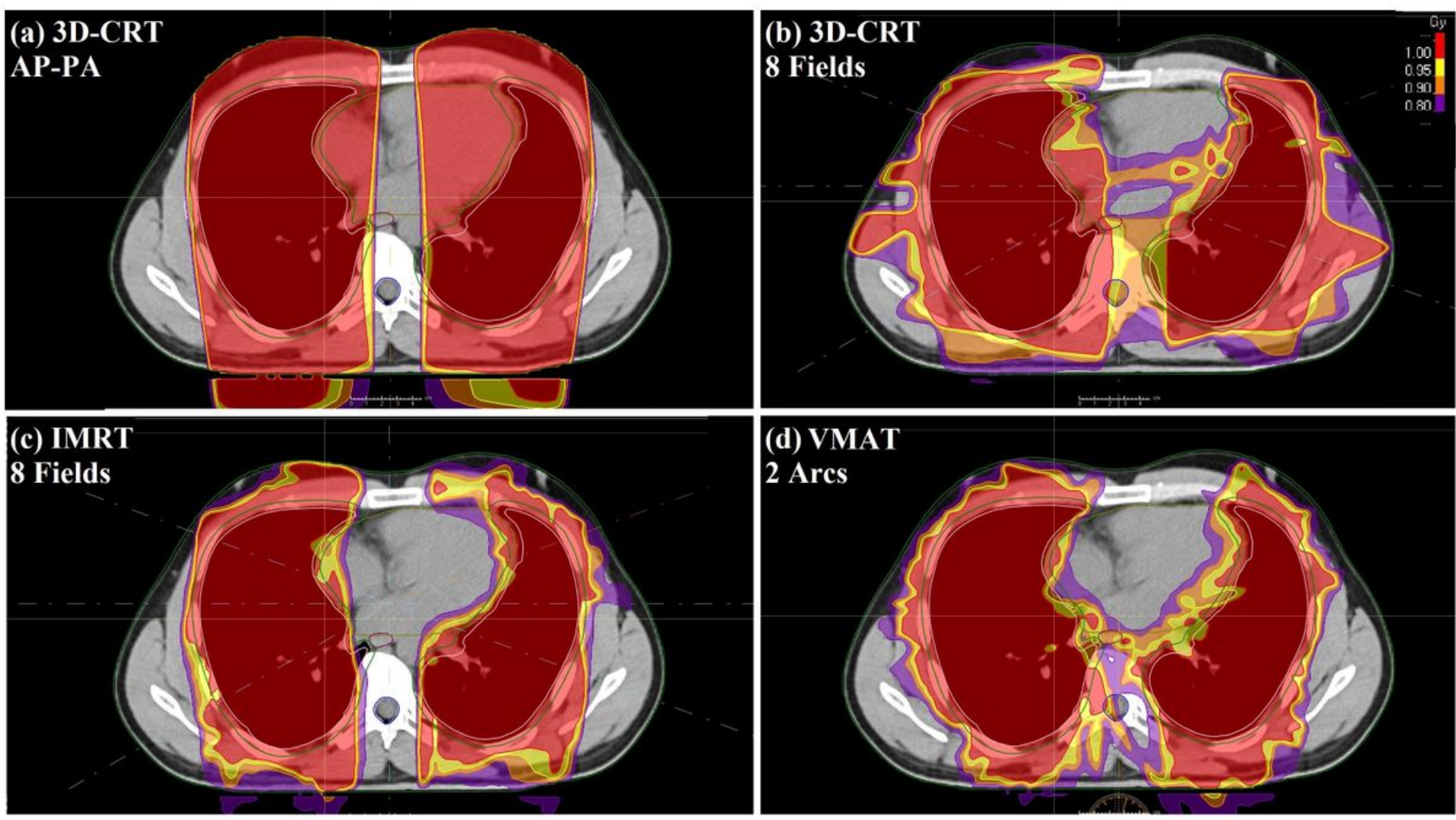

Figure 2 
dose distribution of whole lung LDRT with different delivery techniques and 1Gy prescription for a sample patient in one slice. (a): Dose distribution for 3D-CRT AP-PA technique. (b): Dose distribution for 3D-CRT with 8 coplanar fields technique. (c): Dose distribution for IMRT technique. (d): Dose distribution for VMAT technique.
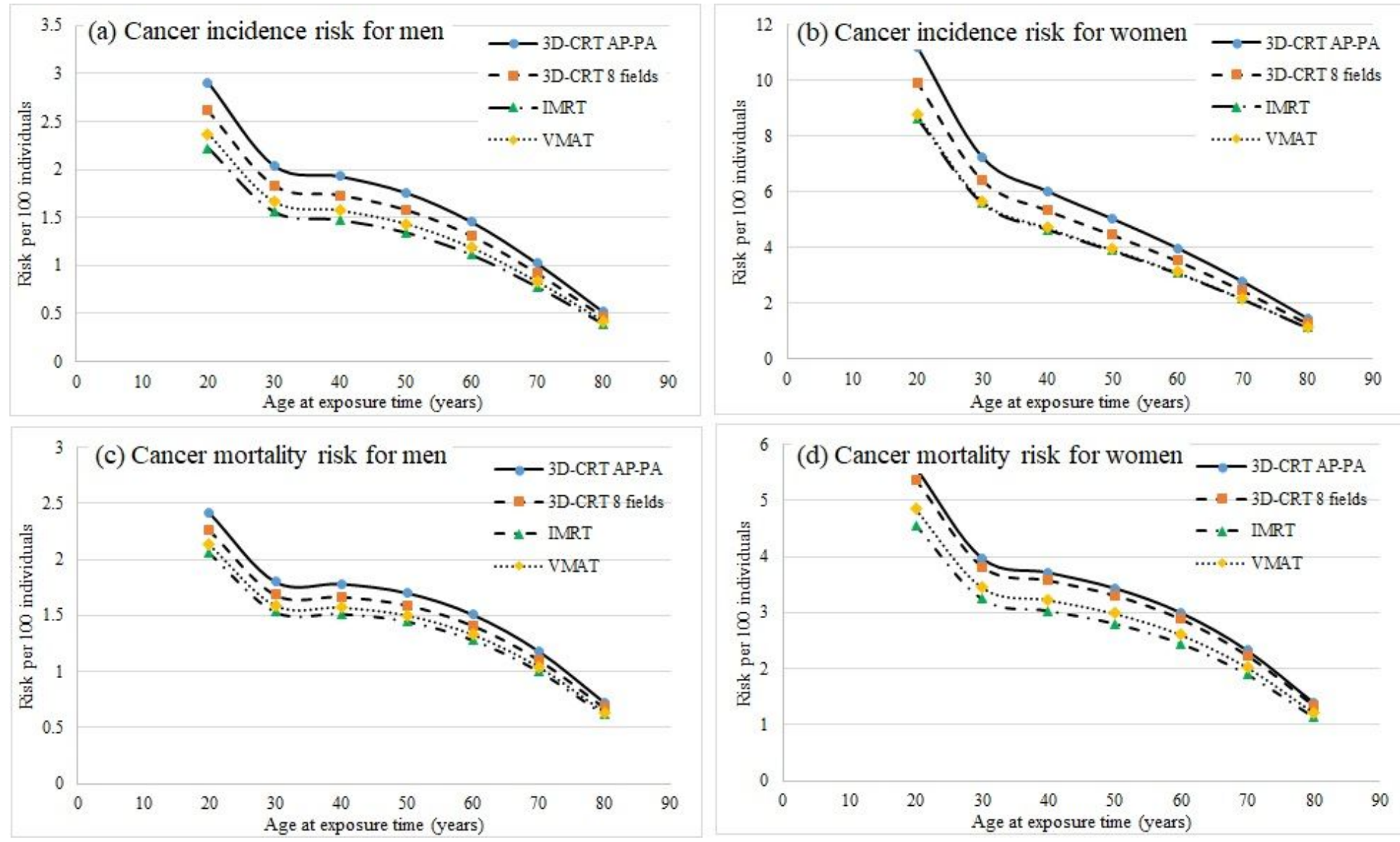

\section{Figure 3}

The total radiation induced lifetime cancer incidence and mortality risks for patients undergoing whole lung LDRT with different radiotherapy techniques at different ages. (a): Cancer incidence risks for male patients at different ages. (b): Cancer incidence risks for female patients at different ages. (c): Cancer mortality risks for male patients at different ages. (d): Cancer mortality risks for female patients at different ages. 